\title{
Numerical modelling of convection-diffusion-adsorption problems in 1D using dynamical discretization
}

\author{
J. Kačur ${ }^{\mathrm{a}}$, B. Malengier ${ }^{\mathrm{b}}$, E. Trojakováa \\ ${ }^{a}$ Faculty of Mathematics, Physics and Informatics, Comenius University Bratislava, Slovakia \\ ${ }^{b}$ Department of Mathematical Analysis, Research Group NfaM ${ }^{2}$, Ghent University, Galglaan 2, B-9000 Ghent, Belgium
}

\begin{abstract}
Two candidates for optimal numerical methods for highly nonlinear partial differential equations are compared. The chosen methods are characterized by their speed, accuracy and simplicity of formulation. They can be easily formulated in high level scripting languages like MATLAB or python and are suited for practical implementation. The setting is that of $1 \mathrm{D}$ general convection-diffusion-adsorption-reaction systems, a setting of high relevance in chemical and groundwater engineering. First, the mathematical model is numerically solved by the method of lines (MOL) using a space discretization with moving grid points (r-adaptivity). The advantage is that with minimal grid points one captures the sharp fronts of the solution which can arise due to a strong adsorption. A large variety of isotherms can be included in the adsorption model for both equilibrium and non-equilibrium modes. In the second method, the mathematical model is approximated using interface modelling. However, this method is only applicable for adsorption in equilibrium mode. The numerical efficiency of the methods is discussed and the obtained numerical results are compared to determine their optimal use. Both methods are very suitable for solving inverse problems in practical implementations, as they are robust and fast.
\end{abstract}

Key words: moving grid points, method of lines, equilibrium adsorption, non-equilibrium adsorption, convective transport, diffusion

\section{Introduction}

Contaminant transport in porous media is modelled (see $[1,2,3]$ among others) by a system of PDE's in the following form:

$$
\begin{array}{r}
\theta \partial_{t} C+\rho \partial_{t} \Psi_{e}(C)+\nabla(\theta \mathbf{v} C)-\nabla(\theta \mathbf{D} \nabla C)+\rho \partial_{t} S=0 \\
\partial_{t} S=\kappa\left(\Psi_{n}(C)-S\right)
\end{array}
$$

where $C$ is the contaminant concentration, $\theta, \rho$ are porosity and specific density of the porous media, $\mathbf{v}$ is the solvent flow in which the contaminant is soluted, and $\Psi_{e}, \Psi_{n}$ are adsorption isotherms in the equilibrium and non-equilibrium mode, respectively. The matrix D represents the dispersion. System (1) must be completed by initial and boundary conditions which will be specified below. The adsorption phenomenon is characterized by an adsorption isotherm - a function that relates the amount of adsorbed material to its amount in the solvent when the equilibrium state is reached. Different adsorbate-adsorbent pairs can have different isotherms. The most common isotherms $\left(\Psi_{e}, \Psi_{n}\right)$ used in practice are:

- Linear isotherm $-\Psi(C)=a C$

- Freundlich isotherm $-\Psi(C)=a C^{p}$

- Langmuir isotherm $-\Psi(C)=\frac{a C}{1+b C}$

Email addresses: kacur@fmph.uniba.sk (J. Kačur), bm@cage.UGent.be (B. Malengier), trojakova@fmph.uniba.sk (E. Trojaková) URL: http://cage.ugent.be/ bm/ (B. Malengier)

Preprint submitted to Chemical Engineering Science 
- Mixed F-L isotherm - $\Psi(C)=\frac{a C^{p}}{1+b C^{p}}$

- Lindstrom-Van Genuchten isotherm - $\Psi(C)=a C e^{-2 b \Psi(C)}$.

If $\kappa \rightarrow \infty$ (case of equilibrium adsorption mode), then $S=\Psi_{n}(C)$ and we can add $\partial_{t} \Psi_{n}(C)$ to the parabolic term. Thus, $\partial_{t} \Psi_{e}(C)$ in (1) represents the adsorption part in equilibrium mode. The porous medium can be a composition of various materials. That means that while some compounds adsorb the contaminant in equilibrium mode, others may adsorb in non-equilibrium mode or need not to adsorb at all. Thus, (1) represents the transport of contaminant in porous media with (reversible) adsorption in equilibrium and non-equilibrium mode. If the term $\partial_{t} S$ in (1) is of the form

$$
\partial_{t} S=\kappa \max \left(0, \Psi_{n}(C)-S\right)
$$

then we have irreversible adsorption.

As many chemical and groundwater engineering laboratory experiments are designed so that the problem reduces to a $1 \mathrm{D}$-flow problem, Eq. (1) is of great relevance already in its $1 \mathrm{D}$ form. The adsorption process can be a side effect that one wants to include in the models (eg separation of chemicals), or the experiment can aim to determine the hydrodynamical and geochemical data included in $\mathbf{D}, \Psi_{e}$ and $\Psi_{n}$. These can be found by the solution of the corresponding inverse problems. Precise and highly efficient numerical solution methods for the direct problem (1) are of extreme importance when dealing with inverse problems.

Although we consider the 1D form of (1) only, the application of the methods discussed in this paper is not limited to 1D experiments. For example, some parameters must be realized in-situ in groundwater engineering. One possibility is the use of a dual-well (an injection and extraction well creating steady state flow), which leads to several simplifications. One of this is that the setting can be transformed (see [4]) to a system of 1D problems of type (1).

Main goal of the paper is the construction of precise and efficient numerical methods to solve 1D problems of the type (1). Several complications arise. If an equilibrium adsorption in (1) (i.e., $\left.\Psi_{n}(s) \equiv 0\right)$ is considered with $\Psi(C)=\frac{a C^{p}}{1+b C^{p}}$ and $0 \leq p<1$, then the solution may obtain the profile of a damped travelling wave with sharp front, which is difficult to resolve in many numerical methods. This phenomenon is furthermore strengthened when an irreversible adsorption is considered combined with an initial concentration profile that is a sharp pulse. Last but not least, the numerical difficulties increase greatly when the problem is convection dominated. From a practical point of view, increasing the flow rhythm in the experiments results in a reduced experiment time. All this suggests that numerical solutions of (1) benefit from the use of methods optimized for the setting, instead of generic codes.

There are many numerical methods devoted to the solution of these type of problems -see, e.g., [2]. One may use types of "up winding" or regularizations (see $[5,6,7]$ ), or operator splitting (see $[4,8,9,10]$ ) and interface modelling (see [11]). The "up winding" or regularizations introduce additional numerical dispersion which dampens the sensitivity of the solution on model parameters. In $[4,8,9,10]$ a splitting method has been used, applied to diffusion, convection and adsorption, and a semi-analytical solution has been found for nonlinear convection in the case of Langmuir and Freundlich isotherms. Dealing with sharp fronts, one should prefer methods that can accurately track the fronts. This is achieved with automatic grid refinement, moving meshes, or interface modelling. Although interface modelling seems the logical choice, the interface evolution model is required. For the non-equilibrium adsorption model in (1) nothing is known about the existence of the interface (which is the boundary of the support of the solution). On the other hand, the drawback of standard grid refinement is that grid projections must be performed.

We construct numerical methods for (1) based on MOL (Method of lines, only a space discretization is done) leading to an ODE system. The advantage of such an approach is that powerful ODE solvers with error control can be used (see however Remark 2) which are readily available in high level scripting languages (matlab, python-scipy, ...). In the first method, developed in $[12,13]$ (see also references therein) this ODE-system is obtained using moving grid points. The main advantage is that the sharp fronts of the solution are captured by automatic adaptivity of moving grid points and the corresponding ODE-solver does not need to be restarted after the time evolution. Also no grid projections are needed. This method is presented in Section 2 as method I.

In Section 3 method II is developed. It can only be applied to adsorption in equilibrium mode, solving the corresponding ODE system using moving grid points which follow the movement of the interface. Method II is based on interface modelling. In the case of adsorption in equilibrium mode $\left(\Psi_{n} \equiv 0\right)$ and with the adsorption isotherm $\Psi_{e}$ satisfying $\Psi_{e}^{\prime}(0)=0$, the solution has an interface (boundary of the support of the solution) provided the initial profile 
has a bounded support. For that case, in [11] an efficient numerical approximation was proposed using moving grid points following the movement of the interface (additional assumptions on $\Psi_{e}$ are needed).

We shall compare these methods on the solution of the model problem (1) in Section 4, as well as with a known analytical solution for a linear problem and with a solution obtained over a very fine grid. The suitability of the methods will be discussed.

We begin by restating the problem in one dimension and in dimensionless variables. For simplicity we assume the one dimensional velocity $v=V \equiv$ const $>0$ and also the diffusion $D$ to be constant. We set $\theta=1$, and we denote $F(C):=C+\rho \Psi_{e}(C)$. We assume a typical length scale of the experiment of $l$ (i.e. sample size between $l$ and $10 l$ ) and concentration values lower than a maximum $\tilde{C}_{0}$. Denoting the dimensional variables by $(\tilde{x}, \tilde{t}, \tilde{C}, \ldots)$, we define a dimensionless counterpart as $f=\tilde{f} / f_{\star}$, where $f_{\star}$ is a characteristic value for the variable $\tilde{f}$. We choose the following characteristic values $x_{\star}=l, v_{\star}=V, t_{\star}=1 / \kappa_{\star}=l / V$ and $C_{\star}=S_{\star}=\tilde{C}_{0}$. Equation (1) then reads in dimensionless form

$$
\begin{array}{r}
\partial_{t} F(C)+\partial_{x} C-P e^{-1} \partial_{x}^{2} C+\rho \partial_{t} S=0 \\
\partial_{t} S=\kappa\left(\Psi_{n}(C)-S\right) .
\end{array}
$$

Two parameters govern the interaction between convection, diffusion and reaction: the Peclet number $P e=l V / D$ indicating the relation between convection and diffusion, and the dimensionless reaction rate $\kappa=\tilde{\kappa} t_{\star}$ indicating the speed of the reaction compared with the speed of convection over a typical length of a sample.

\section{Solution method I: Moving gridpoints}

Together with (2) we consider (in 1D) the initial and boundary conditions

$$
C(x, 0) \equiv 0, \quad S(x, 0) \equiv 0 ; \quad C(0, t)=C_{0}(t) .
$$

We also assume that the solution is so that the concentration does not reach a certain $x=L$, implying that the boundary condition has no influence, allowing us to consider $C(L, t)=0$. The value $C_{0}(t)=1$ is used and at a prescribed time it is switched to $C_{0}(t)=0$. Consider a space discretization with moving grid points

$$
0=x_{0}<x_{1}(t)<\ldots<x_{i}(t)<\ldots x_{N}=L,
$$

which are not yet determined. Let $C\left(x_{i}(t), t\right)$ be the solution along the curve $\left(x_{i}(t), t\right)$. Rewrite (2) in the form (Lagrange coordinates)

$$
\begin{aligned}
& \frac{d}{d t} F\left(C\left(x_{i}(t), t\right)\right)-F^{\prime}\left(C\left(x_{i}(t), t\right)\right) \dot{x}_{i}(t) \partial_{x} C\left(x_{i}(t), t\right) \\
& =\quad-\partial_{x} C\left(x_{i}(t), t\right)+P e^{-1} \partial_{x}^{2} C\left(x_{i}(t), t\right) \\
& \quad-\rho \kappa\left(\Psi_{n}\left(C\left(x_{i}(t), t\right)\right)-S\right), \\
& \begin{aligned}
& \frac{d}{d t} S\left(x_{i}(t), t\right)-\dot{x}_{i}(t) \partial_{x} S\left(x_{i}(t), t\right) \\
&= \kappa\left(\Psi_{n}\left(C\left(x_{i}(t), t\right)\right)-S\right) .
\end{aligned}
\end{aligned}
$$

Apply now the space discretization of the derivatives and denote by $y_{i}(t)$ an approximation of $C\left(x_{i}(t), t\right)$ and by $w_{i}(t)$ an approximation of $S\left(x_{i}(t), t\right)$. The derivatives $\partial_{x} C, \partial_{x}^{2} C$ and $\partial_{x} S$ are approximated at the grid point $x_{i}$ by a three point approximation using the Lagrange polynomial $\mathcal{L}\left(z ; y_{i}\right)$ of the second order crossing the points

$$
\left(x_{i-1}(t), y_{i-1}(t)\right),\left(x_{i}(t), y_{i}(t)\right),\left(x_{i+1}(t), y_{i+1}(t)\right) .
$$

Then,

$$
y(t):=\left(y_{1}(t), \ldots, y_{N-1}(t)\right)
$$

and

$$
w(t):=\left(w_{0}(t), w_{1}(t), \ldots, w_{N-1}(t)\right)
$$


are the numerical approximation of (2), (3), determined by the ODE-system

$$
\begin{gathered}
F^{\prime}\left(y_{i}\right)\left[\dot{y}_{i}-\left.\dot{x}_{i} \frac{d \mathcal{L}\left(z ; y_{i}\right)}{d z}\right|_{z=x_{i}}\right]=-\left.\frac{d \mathcal{L}\left(z ; y_{i}\right)}{d z}\right|_{z=x_{i}} \\
\quad+\left.P e^{-1} \frac{d^{2} \mathcal{L}\left(z ; y_{i} i\right.}{d z^{2}}\right|_{z=x_{i}}-\rho \kappa\left(\Psi_{n}\left(y_{i}\right)-w_{i}\right), \\
\dot{w}_{i}-\left.\dot{x}_{i} \frac{d \mathcal{L}\left(z ; w_{i}\right)}{d z}\right|_{z=x_{i}}=\kappa\left(\Psi_{n}\left(y_{i}\right)-w_{i}\right),
\end{gathered}
$$

for $i=1, \ldots, N-1$, where $y_{0}=C_{0}$ and $x(t):=\left(x_{1}(t), \ldots, x_{N-1}(t)\right)$ are yet unknown. To determine $x(t)$ we follow [12] where the density of moving grid points automatically follows the high spatial activity of the solution. For this purpose we define the monitoring function

$$
m \equiv m(x ; C, S):=\sqrt{\gamma+\delta\left(\partial_{x} C\right)^{2}+(1-\delta)\left(\partial_{x} S\right)^{2}},
$$

with $\gamma>0, \delta \in[0,1]$. We replace $C, S$ by their approximations $y, w$ and use central differences for the space derivative. By $m_{i}$ we denote the approximation of $m$ over the interval $\left[x_{i-1}, x_{i}\right]$, given by the formula

$$
m_{i}:=\sqrt{\gamma+\delta\left(\frac{y_{i}-y_{i-1}}{x_{i}-x_{i-1}}\right)^{2}+(1-\delta)\left(\frac{w_{i}-w_{i-1}}{x_{i}-x_{i-1}}\right)^{2}},
$$

for $i=1, \ldots, N-1$. Note that the first derivatives can also be approximated by the three point approximation mentioned above.

Denote

$$
\alpha_{i}(t):=x_{i}(t)-x_{i-1}(t), \quad \text { for } i=1, \ldots, N,
$$

then

$$
n_{i}:=\frac{1}{\alpha_{i}}, \quad i=1, \ldots, N,
$$

represent the densities of grid points. To avoid collapsing, or crossing of grid points, "anti-diffused" densities $\tilde{n}_{i}$ are introduced (see [12]):

$$
\begin{aligned}
\tilde{n}_{1} & :=n_{1}-\mu\left(n_{2}-n_{1}\right) \\
\tilde{n}_{i} & :=n_{i}-\mu\left(n_{i+1}+n_{i-1}-2 n_{i}\right), i=2, \ldots, N-1 \\
\tilde{n}_{N} & :=n_{N}-\mu\left(n_{N-1}-n_{N}\right)
\end{aligned}
$$

where $\mu=k(k+1)$ and $k>0$ (we take $k \in(1,2)$ ) is a parameter. The main rule for the redistribution of grid points is given by the system

$$
\frac{\tilde{n}_{i-1}}{m_{i-1}}=\frac{\tilde{n}_{i}}{m_{i}}, i=2, \ldots, N,
$$

which represent an equidistribution of grid points along the arc of the solution. If $\tilde{n}$ is determined by (9), then $n$ computed from (8) satisfies

$$
\frac{k}{k+1} \leq \frac{n_{i-1}}{n_{i}} \leq \frac{k+1}{k} .
$$

Finally, to guarantee temporal grid-smoothing, the algebraic system (9) is replaced by the ODE system

$$
\frac{\tilde{n}_{i-1}+\tau \dot{\tilde{n}}_{i-1}}{m_{i-1}}=\frac{\tilde{n}_{i}+\tau \dot{\tilde{n}}_{i}}{m_{i}}, \quad i=2, \ldots, N,
$$

where $\tau=$ const, $>0$. Those are the governing equations for the moving grid points. Rewriting this system in terms of $x(t)$ using (8), (6), (7) and

$$
\dot{n}_{i}(t)=-\frac{\dot{\alpha}_{i}(t)}{\alpha_{i}^{2}(t)}, \quad \dot{\alpha}_{i}(t)=\dot{x}_{i}(t)-\dot{x}_{i-1}(t),
$$


and substituting in (11), yields the desired governing mathematical model for the moving grid points (see [12])

$$
\begin{gathered}
-\tau\left[\frac{\mu}{m_{i} \alpha_{i-1}^{2}}\right] \dot{x}_{i-2}+\tau\left[\frac{\mu}{m_{i+1} \alpha_{i}^{2}}+\frac{1+2 \mu}{m_{i} \alpha_{i}^{2}}+\frac{\mu}{m_{i} \alpha_{i-1}^{2}}\right] \dot{x}_{i-1}- \\
\tau\left[\frac{\mu}{m_{i+1} \alpha_{i}^{2}}+\frac{1+2 \mu}{m_{i+1} \alpha_{i+1}^{2}}+\frac{1+2 \mu}{m_{i} \alpha_{i}^{2}}+\frac{\mu}{m_{i} \alpha_{i+1}^{2}}\right] \dot{x}_{i}+ \\
\tau\left[\frac{\mu}{m_{i+1} \alpha_{i+2}^{2}}+\frac{1+2 \mu}{m_{i+1} \alpha_{i+1}^{2}}+\frac{\mu}{m_{i} \alpha_{i+1}^{2}}\right] \dot{x}_{i+1}-\tau\left[\frac{\mu}{m_{i+1} \alpha_{i+2}^{2}}\right] \dot{x}_{i+2}= \\
\frac{1}{m_{i+1}}\left[-\frac{\mu}{\alpha_{i+2}}+\frac{1+2 \mu}{\alpha_{i+1}}-\frac{\mu}{\alpha_{i}}\right]-\frac{1}{m_{i}}\left[-\frac{\mu}{\alpha_{i+1}}+\frac{1+2 \mu}{\alpha_{i}}-\frac{\mu}{\alpha_{i-1}}\right]
\end{gathered}
$$

for $2 \leq i \leq N-2$, where $\dot{x}_{0}=0, \dot{x}_{N}=0$. We complete this system with the following equations for $i=1$ and $i=N-1$

$$
\begin{gathered}
-\tau\left[\frac{1+\mu}{m_{1} \alpha_{1}^{2}}+\frac{1+2 \mu}{m_{2} \alpha_{2}^{2}}+\frac{\mu}{m_{1} \alpha_{2}^{2}}+\frac{\mu}{m_{2} \alpha_{1}^{2}}\right] \dot{x}_{1}+ \\
\tau\left[\frac{\mu}{m_{1} \alpha_{2}^{2}}+\frac{1+2 \mu}{m_{2} \alpha_{2}^{2}}+\frac{\mu}{m_{2} \alpha_{3}^{2}}\right] \dot{x}_{2}-\tau\left[\frac{\mu}{m_{2} \alpha_{3}^{2}}\right] \dot{x}_{3} \\
=\frac{1}{m_{2}}\left[-\frac{\mu}{\alpha_{1}}+\frac{1+2 \mu}{\alpha_{2}}-\frac{\mu}{\alpha_{3}}\right]-\frac{1}{m_{1}}\left[\frac{1+\mu}{\alpha_{1}}-\frac{\mu}{\alpha_{2}}\right], \\
-\tau\left[\frac{\mu}{m_{N-1} \alpha_{N-2}^{2}}\right] \dot{x}_{N-3}+\tau\left[\frac{\mu}{m_{N-1} \alpha_{N-2}^{2}}+\right. \\
\left.\tau\left[\left(\frac{1+2 \mu}{m_{N-1}}+\frac{\mu}{m_{N-1}}\right)+\frac{\mu}{m_{N}}\right) \alpha_{N-1}^{-2}+\left(\frac{1+\mu}{m_{N}}+\frac{\mu}{m_{N-1}}\right) \alpha_{N}^{-2}\right] \dot{x}_{N-1}= \\
\frac{1}{m_{N}}\left[\frac{1+\mu}{\alpha_{N}}-\frac{\mu}{\alpha_{N-1}}\right]-\frac{1}{m_{N-1}}\left[\frac{1+2 \mu}{\alpha_{N-1}}-\frac{\mu}{\alpha_{N}}-\frac{\mu}{\alpha_{N-2}}\right]
\end{gathered}
$$

The final ODE system for our approximate solution is given by (5),(12), (13) and (14). Denoting $z(t):=(y(t)$, $w(t), x(t))$ allows to rewrite our complex ODE system in the compact form

$$
M(t, z(t)) \dot{z}=g(t, z(t))
$$

where the matrix $M$ and the vector $g$ are uniquely determined by (5), (12), (13) and (14). This system has to be completed by an initial vector $z_{0}=z(0)$, generated by means of initial conditions. We solve the system with an ODE solver for stiff systems. In the numerical experiments we use the solver daspk ${ }^{1}$, but also "ode $15 \mathrm{~s}$ " from the toolbox of MATLAB could be used.

Remark 1. The distribution of initial grid points $x(0)$ also takes equidistance along the initial profile $C(x, 0)$, resp. $S(x, 0)$, into account. To avoid the singularity at the left boundary and to remove instabilities at the initial phase of the solution, we have to regularize the initial vector $y(0)=0$ and take it positive in a few of the first grid points (taking the boundary condition into consideration). If the adsorption problem is degenerate (i.e. $\left.\Psi_{e}^{\prime}(0)=\infty\right)$, then system (15) degenerates and further regularization is needed. We regularize replacing $1 / F^{\prime}(C)$ by $\max \left(\varepsilon, 1 / F^{\prime}(C)\right.$ ) with a sufficiently small $\varepsilon>0$. This slight regularization does not change the solution significantly, which can be justified numerically.

\footnotetext{
${ }^{1}$ See http://www.netlib.org/ode, adapted for use in python-scipy via odes scikit, http://projects.scipy.org/scipy/scikits/ browser/trunk/odes - http://cage.ugent.be/ bm/progs.html
} 
Remark 2. The ODE solvers used provide error control with respect to the ODE system, here (15). This error control is not directly related to the original PDE (2) as the space discretization is not taken into account. It applies to the ODE system only. Other solution methods like e.g. Rothe's method are better suited to control the errors, especially in the space variable, since it allows the change of gridpoints along time based on an error estimator.

\section{Solution method II: Interface modelling.}

This method is based on the existence of the interface (the boundary of the support of the solution) which for our mathematical model (2) in $1 \mathrm{D}$ exists for adsorption in equilibrium mode $\left(\Psi_{n}(s) \equiv 0\right)$ when $\Psi_{e}^{\prime}(0)=\infty$. In the case of conditions (3), the interface $s(t)$ starts at $x=0$ and when $V \geq 0$ moves to the right. Interface modelling solves the problem only in the domain $(0, s(t))$ since $C(x, t)=0$ for $x \geq s(t)$. It uses moving grid points $x_{i}(t)=\xi_{i} s(t)$ for $i=1, \ldots, N-1$, where

$$
0=\xi_{0}<\xi_{1}<\ldots<\xi_{i}<\ldots \xi_{N}=1
$$

are fixed gridpoints over the interval $[0,1]$. As before, we apply the MOL method and arrive at the governing ODE system (4) without the non-equilibrium part $(\kappa=0)$. In this case the governing ODE system for the moving gridpoints consists of one ODE for $\dot{s}(t)$. Therefore, the construction of the mathematical model for $s(t)$ is crucial for this method. Following [11], this model can be obtained in cases when the equilibrium adsorption is asymptotically polynomial $\Psi_{e}(s) \approx s^{q}$ for $s \searrow 0$. We present here the mathematical model in the case of a Freundlich and a mixed F-L isotherm $\Psi(C)=\frac{a C^{p}}{1+b C^{p}}$ with $p \in(0,1)$, which represents a large scale of frequently used isotherms in practice. We have

$$
\dot{s}(t)=-\frac{P e^{-1}}{\rho a(1-p)} \partial_{x} v \quad \text { for } x=s(t), C=v^{\beta}, \beta=\frac{1}{1-p} .
$$

Here, $\partial_{x} v \neq 0$ for $x=s(t)$. Apply the transformation $C=v^{\beta}, \beta=\frac{1}{1-p}$, also in (5) to get

$$
\begin{aligned}
& F^{\prime}\left(v^{\beta}\left(x_{i}(t), t\right)\right) \partial_{t} v\left(x_{i}(t), t\right)=-\partial_{x} v\left(x_{i}(t), t\right)+ \\
& P e^{-1} \partial_{x}^{2} v\left(x_{i}(t), t\right)+P e^{-1} \frac{p}{(1-p) v\left(x_{i}(t), t\right)}\left(\partial_{x} v\left(x_{i}(t), t\right)\right)^{2} \\
& \quad+F^{\prime}\left(v^{\beta}\left(x_{i}(t), t\right)\right) \dot{x}_{i}(t) \partial_{x} v\left(x_{i}(t), t\right) .
\end{aligned}
$$

Next, we use the transformation $\xi=\frac{x}{s(t)}$ where $\xi \in[0,1]$, with the grid points $\left\{\xi_{i}\right\}_{i=0}^{N}$, and consequently $x_{i}(t)=\xi_{i} s(t)$. Denoting $Y_{i}(t) \approx v\left(\xi_{i} s(t), t\right),(17)$ is approximated by the following ODE-system

$$
\begin{aligned}
\dot{Y}_{i}=\frac{1}{F^{\prime}\left(Y_{i}^{\beta}\right)}\left[-\left.\frac{1}{s} \frac{d \mathcal{L}\left(\xi ; Y_{i}\right)}{d \xi}\right|_{\xi=\xi_{i}}+\left.\frac{P e^{-1}}{s^{2}} \frac{d^{2} \mathcal{L}\left(\xi ; Y_{i}\right)}{d \xi^{2}}\right|_{\xi=\xi_{i}}\right. & \\
& \left.+\frac{P e^{-1} p}{(1-p) s^{2} Y_{i}}\left(\left.\frac{d \mathcal{L}\left(\xi ; Y_{i}\right)}{d \xi}\right|_{\xi=\xi_{i}}\right)^{2}\right]+\left.\xi_{i} \frac{I(Y, p)}{s} \frac{d \mathcal{L}\left(\xi ; Y_{i}\right)}{d \xi}\right|_{\xi=\xi_{i}},
\end{aligned}
$$

which is completed by the governing interface equation

$$
\dot{s}(t)=-\left.\frac{P e^{-1}}{\rho a(1-p)} \frac{d^{2} \mathcal{L}(\xi ; 1)}{d \xi^{2}}\right|_{\xi=1}=: I(Y, p),
$$

where $\mathcal{L}(\xi ; 1)$ is the Lagrange polynomial of the second order crossing the points $\left(\xi_{N-2}, Y_{N-2}\right),\left(\xi_{N-1}, Y_{N-1}\right)$ and $(1,0)$, since the solution $C$ has zero value at $x=s(t)$. ODE-system (18), (19) is again stiff, but causes no problem for the used ODE-solver. Then, $y_{i}(t):=Y_{i}^{\beta}(t)$ is a numerical approximation of $C\left(x_{i}(t), t\right)$ with $x_{i}(t)=\xi_{i} s(t)$ for $i=1, \ldots, N-1$.

Remark 3. This method is suitable for solutions of semi-wave type with sharp fronts at the interface. This is the case for the mathematical model with adsorption in equilibrium mode with the property $\Psi_{e}^{\prime}(0)=\infty$. A sharp front at the interface can appear and continue throughout time. To apply the method we choose a non-uniform space discretization $\left\{\xi_{i}\right\}$ with increasing density in the neighborhood of the point $\xi=1$, which corresponds to the interface $x=s(t)$. The advantage is that the dimension of the ODE-system is small since we have only one additional ODE equation to describe the moving grid points. The numerical experiments and comparison with the previous methods is presented in Section 4. 
Remark 4. If the adsorption in equilibrium mode is not degenerate (i.e. $\Psi_{e}^{\prime}(0)<\infty$ ), then there is no interface (the problem is regular). Also in that case we can use interface modelling by considering a modified problem where we replace $\Psi(s)$ by $\Psi\left(s^{p}\right)$ with $p \nearrow 1$. We note that the case $p=1$ is singular for our method II (see (19)) since there exists no interface.

\section{Numerical experiments.}

For most of the problems occurring in practice no solutions in analytical form are known. Therefore, we construct very precise numerical solutions using a high density of grid points and compare those with the results obtained by the presented numerical methods. In the general case of our 1D problem (2), (3) we also construct a numerical solution as in Section 2 (in terms of $y(t), w(t)$ ) with a fixed dense grid instead of a moving grid, and solve it with the ODE-solver. This allows to control the sharp front of the solution. We denote this standard numerical approximation as method III, and compare the efficiency in the experiments mentioned below.

In most experiments the numerical results obtained by different methods with the same model parameters are

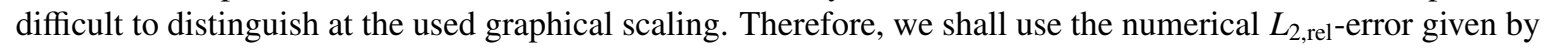

$$
L_{2, \text { rel }}\left(t_{e}\right):=\left\{\frac{\sum_{i=1}^{N}\left(u_{A}\left(x_{i}, t_{e}\right)-u_{B}\left(x_{i}, t_{e}\right)\right)^{2} \Delta x_{i}}{\sum_{i=1}^{N}\left(u_{B}^{2}\left(x_{i}, t_{e}\right) \Delta x_{i}\right.}\right\}^{1 / 2},
$$

where $u_{A}$ and $u_{B}$ are two solutions obtained with the methods $A$ and $B(A, B \in\{I, I I, I I I\})$, and interpolated at the same grid points $\left\{x_{i}\right\}_{i=1}^{N}$. As we use a MOL approach to time integration, we need to set a precision for the ODE solver. We choose an absolute and a relative precision of $10^{-6}$. This precision determines the size of the time steps taken in the time integration.

In the case that an analytical solution is known, we compare a numerical solution $u_{A}$ with the analytical $u_{\text {an }}$ by replacing $u_{B}$ with $u_{a n}$ in (20). An analytical solution is known for the following linear problem :

$$
\begin{gathered}
\partial_{t} C+\partial_{x} C-P e^{-1} \partial_{x}^{2} C+\partial_{t} S=0 \\
\partial_{t} S=\kappa(C-S)
\end{gathered}
$$

with boundary conditions:

$$
C(0, t)=1, \quad \partial_{x} C(L, t)=0
$$

and initial conditions:

$$
C(x, 0)=0, \quad S(x, 0)=0
$$

The analytical solution constructed according to [14] is:

$$
\begin{aligned}
& C(x, t)=\frac{5}{2} J(x, t) e^{-\kappa t} \\
& +\frac{5}{2} \int_{0}^{t} J(x, z) e^{-\kappa t}\left(I_{0}\left(2 \kappa \sqrt{t z-z^{2}}\right)\right. \\
& \left.+\sqrt{\frac{z}{t-z}} I_{1}\left(2 \kappa \sqrt{t z-z^{2}}\right)\right) d z
\end{aligned}
$$

where $I_{0}$ and $I_{1}$ are modified Bessel functions of order zero and one, and

$$
J(x, t)=\frac{1}{2} \operatorname{erfc}\left(\frac{x-t}{\sqrt{4 P e^{-1} t}}\right)+\frac{1}{2} e^{x P e} \operatorname{erfc}\left(\frac{x+t}{\sqrt{4 P e^{-1} t}}\right)
$$

The remaining unknown $S(x, t)$ can be expressed by solving (22). We have to notice here that, though we can obtain an analytical formula for the solution, the evaluation of $C(x, t)$ (and consequently $S(x, t)$ ) requires numerical integration. Note that the numerical cost of such an evaluation is actually comparable with the cost of solving the problem by using a numerical scheme. 


\begin{tabular}{|l|l|l|l|}
\hline$\gamma$ & $\tau$ & time & $\epsilon(12) .10^{2}$ \\
\hline 0.005 & 0.0005 & 6.66 & 0.112 \\
\hline 0.05 & 0.0005 & 3.25 & 0.0406 \\
\hline 0.05 & 0.00005 & 3.53 & 0.0385 \\
\hline
\end{tabular}

Table 1: Comparison of Method I with analytical solution (Exp 1)

\begin{tabular}{|l|l|l|l|l|}
\hline$N$ & time & $\epsilon(4) .10^{2}$ & $\epsilon(8) .10^{2}$ & $\epsilon(12) .10^{2}$ \\
\hline 20 & $1.6 ”$ & 1.68 & 1.47 & 1.27 \\
\hline 40 & $1.4 ”$ & 0.443 & 0.436 & 0.401 \\
\hline 80 & $1.7 ”$ & 0.161 & 0.151 & 0.137 \\
\hline 160 & $2.3 "$ & 0.0784 & 0.0648 & 0.0584 \\
\hline 300 & $3.5 "$ & 0.0572 & 0.0424 & 0.0385 \\
\hline
\end{tabular}

Table 2: Comparison of Method I with analytical solution (Exp 1)

The analytical solution contains a non-equilibrium adsorption term and hence can only be compared with methods I and III. We will start with such a comparison in the first experiment

Experiment 1. First, in this experiment we shall compare the numerical solution obtained by method I (moving grid approximation) with the analytical solution. We use the following model parameters $\kappa=5 / 2, P e^{-1}=0.05, L=$ 10. As Method I depends on some tuning parameters, we first give an overview in Table 1 of the error in comparison to the analytical solution for different values of the parameters. Here $\delta=0.5, k=2$ and 300 grid points are considered. Based on the results we will take for all further experiments with Method I $\gamma=0.05$ and $\tau=0.00005$. Reducing $\tau$ further dramatically increases the computational time.

We now compare Method I and Method III with the analytical solutions. The values of $\epsilon\left(t_{e}\right) \equiv L_{2, r e l}\left(t_{e}\right)$ are presented in Table 2 for different number of grid points $(N)$. Table 3 gives the $\epsilon\left(t_{e}\right)$-error between the numerical solution of method III and the analytical solution.

Tables 2-3 also show the time cost of the computations (here' denotes minutes and" denotes seconds). These are strongly dependent on the linearity of the mathematical model in this experiment.

When discussing the results, one has to keep in mind that the analytical solution is also obtained by numerical approximations and its evaluation is comparable with the numerical computation of the model (with less grid points). Comparing Table 3 with Table 2 shows that the numerical method provides an approximation of the real solution as good as that of the analytical one. Method III is less time consuming in this linear experiment, even when many grid points are used and is comparable with method I, where less grid points are used but the size of the ODE-system is increased by including the moving grid model. Notice that Method I has decreasing errors going from time 8 to 12 , whereas Method III has increasing errors. This is a consequence of the fact that the grid points follow the profile,

\begin{tabular}{|l|l|l|l|l|}
\hline$N$ & time & $\epsilon(4) .10^{2}$ & $\epsilon(8) .10^{2}$ & $\epsilon(12) .10^{2}$ \\
\hline 25 & $<1 "$ & 3.6 & 2.3 & 1.7 \\
\hline 50 & $<1 "$ & 0.924 & 0.541 & 0.397 \\
\hline 100 & $<1 "$ & 0.229 & 0.133 & 0.101 \\
\hline 200 & $<1 "$ & 0.0586 & 0.0329 & 0.0319 \\
\hline 400 & $<1 "$ & 0.018 & 0.0083 & 0.0202 \\
\hline 800 & $<1 "$ & 0.011 & 0.0022 & 0.0189 \\
\hline
\end{tabular}

Table 3: Comparison of Method III with analytical solution (Exp 1) 


\begin{tabular}{|l|l|l|l|l|l|}
\hline$N$ & time & $\epsilon_{I}(8) .10^{2}$ & $\epsilon_{I}(12) .10^{2}$ & $\epsilon_{I I I}(8) .10^{2}$ & $\epsilon_{I I I}(12) .10^{2}$ \\
\hline \multicolumn{5}{|l|}{ Method I } \\
\hline 40 & $6 "$ & 0.851 & 1.072 & 0.919 & 1.129 \\
\hline 80 & $14 ”$ & 0.305 & 0.383 & 0.373 & 0.439 \\
\hline 160 & $37 ”$ & 0.113 & 0.136 & 0.181 & 0.192 \\
\hline 300 & $41 "$ & 0.0481 & 0.0524 & 0.0954 & 0.0918 \\
\hline \multicolumn{7}{|l|}{ Method III } \\
\hline 50 & $4 "$ & 0.597 & 0.510 & 0.570 & 0.484 \\
\hline 100 & $6 "$ & 0.180 & 0.155 & 0.150 & 0.128 \\
\hline 200 & $8 ”$ & 0.0899 & 0.0772 & 0.0537 & 0.0451 \\
\hline 400 & $23 ”$ & 0.0712 & 0.0609 & 0.0311 & 0.0259 \\
\hline
\end{tabular}

Table 4: Comparison of Method I and III to solutions obtained with small grid sizes (Exp 2)

\begin{tabular}{|l|l|l|l|l|l|}
\hline$\epsilon(12) .10^{2}$ & $I$ & $I I$ & $I I I$ & $I_{\mathrm{NE}}$ & $I I I_{\mathrm{NE}}$ \\
\hline \hline$I$ & $16 "$ & $/$ & $/$ & $/$ & $/$ \\
\hline$I I$ & 7.43 & $<1 ”$ & $/$ & $/$ & $/$ \\
\hline$I I I$ & 7.15 & 0.422 & $77^{\prime \prime}$ & $/$ & $/$ \\
\hline$I_{\mathrm{NE}}$ & 4.10 & 3.631 & 3.50 & $122^{\prime \prime}$ & $/$ \\
\hline$I I I_{\mathrm{NE}}$ & 7.43 & 0.883 & 0.111 & 3.43 & $347^{\prime}$ \\
\hline
\end{tabular}

Table 5: Comparison between the method I, II, III (Exp 3)

reducing the grid errors added over time to the solution. In this linear example little can be said about the optimal grid size, although it can be noted that Method I over longer time periods performs as good as Method III with roughly $20 \%$ less grid points. Method I is also more robust when very few grid points are taken. It must be noted that the precision of the time integration (set to $10^{-6}$ ) has a larger influence on the observed error.

Experiment 2. We discuss the efficiency of the introduced methods in the case of nonlinear adsorption. Consider (21) with initial and boundary conditions as in Experiment 1. Replace (22) by the Freundlich type of adsorption

$$
\partial_{t} S=\kappa\left(C^{p}-S\right)
$$

with $p=0.5$. In this case we are not aware of an analytical solution. Here, we consider $L=8$ and time points $T_{e}=8, T_{e}=12$. We apply the methods I and III. Table 4 gives the number of grid points, measured computational time at time $t=12$ and the relative errors $\epsilon(8), \epsilon(12)$, when the solutions are compared to a very fine equidistant grid $\left(N=4000\right.$, error $\left.\epsilon_{I I}\right)$ and fine moving grid $\left(N=720\right.$, error $\left.\epsilon_{I}\right)$.

The obtained results in this experiments show the following. The computational time in the nonlinear adsorption is significantly larger then in the linear case. For higher degeneracy (i.e. smaller value $p$ ), more computing time is needed. Method III with a fixed grid has equal error as the results of Method I for the same number of gridpoints, but is much faster. Hence, for this experiment there is no apparent benefit of using moving grid points and the overhead this entails.

Experiment 3. In this experiment we consider the mathematical model (2) in 1D with $\kappa=0$ and with the initial and boundary conditions (3). We consider $\Psi_{e}(C)=C^{p}$, i,e., $F(C)=C+C^{p}$ which corresponds to adsorption of Freundlich type in equilibrium mode. Set $\rho=1, a=1$ and $P e^{-1}=0.05$ for this experiment. This is the first experiment that allows us to use method II. The result is compared also with method I and III where $\kappa=25$, which represents very quick adsorption kinetics. We expect to be close to the equilibrium adsorption. We take $N=100$ in all the methods, and present in Table 5 the errors between the methods at time level $T_{e}=12$. On the diagonal we present the computing time. When we compare results by the method II, using $N=100$ and $N=40$ grid points, then $\epsilon\left(t_{e}=12\right)=0.00432$.

We conclude that in the problems with an interface, method II is by far the most efficient. The results with the methods II and III almost coincide, whereas method I gives a larger difference. It appears that method I has difficulties with the nonlinearity at the interface, specifically in approximating the correct speed of the interface, leading to a 


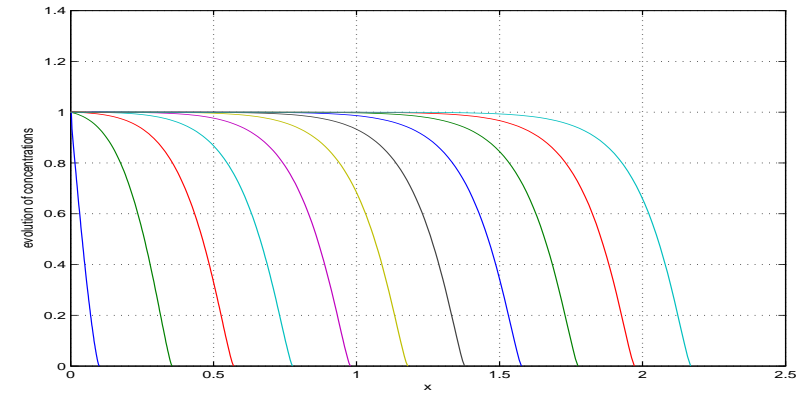

(a) Phase I, $t \in(0,4)$

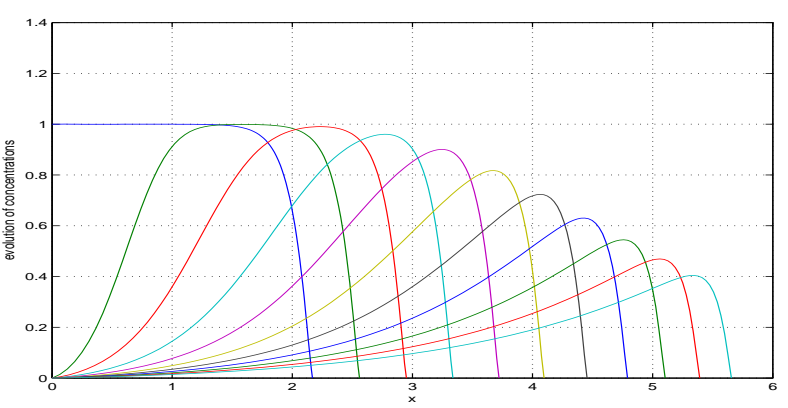

(b) Phase II, $t \in(0,8)$

Figure 1: Method II: $p=0.25, N=100, P e^{-1}=0.05$

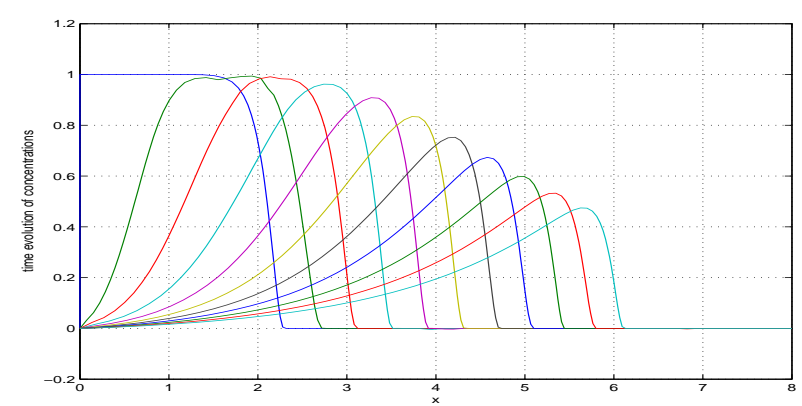

(a) Concentration

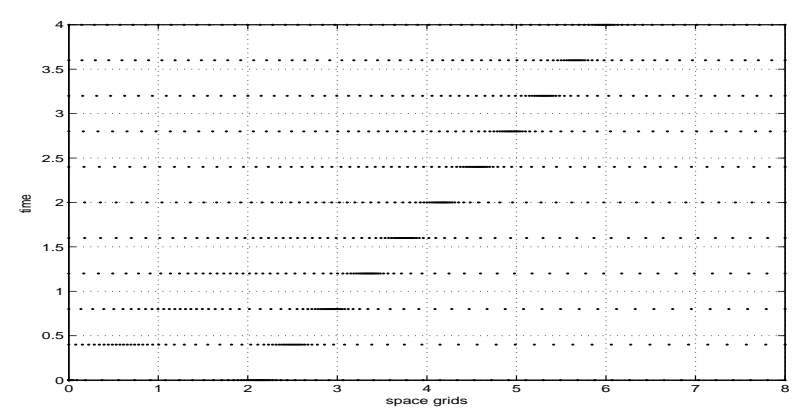

(b) Moving grid points

Figure 2: Method I: phase II, $p=0.25, N=100, P e^{-1}=0.05, t \in(0,8)$

higher error, although the profile is correct. This is due to the discontinuity in the monitor at the interface, combined with $F^{\prime}(y) \longrightarrow \infty$ there. A regularization has been done. We have chosen to take a low initial concentration profile at $t=0$.

The non-equilibrium approximations of the equilibrium adsorption perform reasonably well. In the case of a fixed grid there is however a large increase of computation time. Hence, Method III is not advisable when $\kappa$ is large.

Experiment 4. In this experiment we present several concentration profiles showing the result of different equilibrium and non-equilibrium sorption isotherms. We begin with solutions obtained by method II. We use $p=0.25$ and $P e^{-1}=0.05$, where Freundlich adsorption isotherms $\Psi_{e}(s)=s^{p}$ and $\Psi_{n}(s)=0$ are used. This is a sufficiently degenerate case of adsorption to stress the methods. In Fig. 1(a) we present the time evolution of the solution in the time interval $(0,4)$ over 10 equidistant time steps (phase I). The solution at $t=4$ will be the initial profile of the same problem, but with the homogeneous Dirichlet boundary condition at $x=0$. We solve this problem along the time interval $(0,8)$ (phase II). The results are drawn in Fig. 1(b). The same problem is approximated with a nonequilibrium adsorption problem, where $\Psi_{e}(s)=0, \Psi_{n}(s)=s^{0.25}$ and $\kappa=15$. We solve it with the method I using 60 grid points. In Fig. 2(a) we present the time evolution of phase II corresponding to Fig. 1(b). In Fig. 2(b) the time evolution of moving grid points, corresponding to the same time points of the concentration profiles in Fig. 2(a), is shown. The regularization of method I used here consists in introducing an initial profile which is non-zero in the first space interval $(0, L / N)$, where $N$ is the number of grid points and $L$ is the length of the computational domain. This is achieved by inserting 10 equidistantly distributed grid points into the first space interval, which allows to control the sharp front. Also at the start of phase II we insert 10 additional grid points into the first space interval to control the abrupt jump in boundary condition. We can see that the grid points follow the movement of sharp front of the solution. Method II needs for this problem less than 5 seconds, which emphasizes its efficiency.

Using equilibrium adsorption with $\Psi_{e}(s)=s^{0.25}, \Psi_{n}(s)=0$ and $\kappa=0$ in method I leads to higher singularity and loss of stability in solving the corresponding ODE-system. To overcome this, an initial profile over the entire domain 


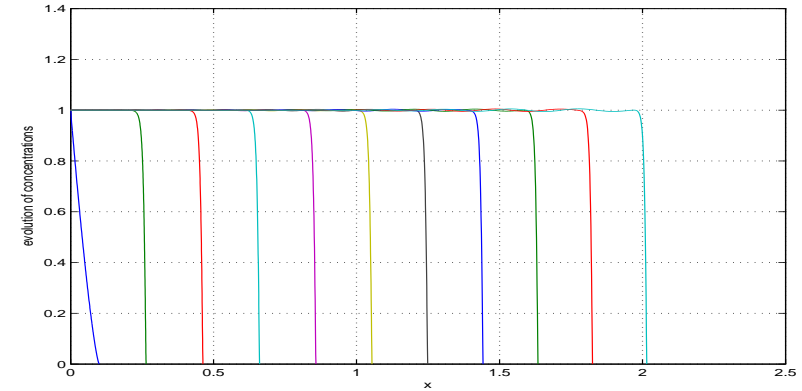

(a) Phase I, $t \in(0,4)$

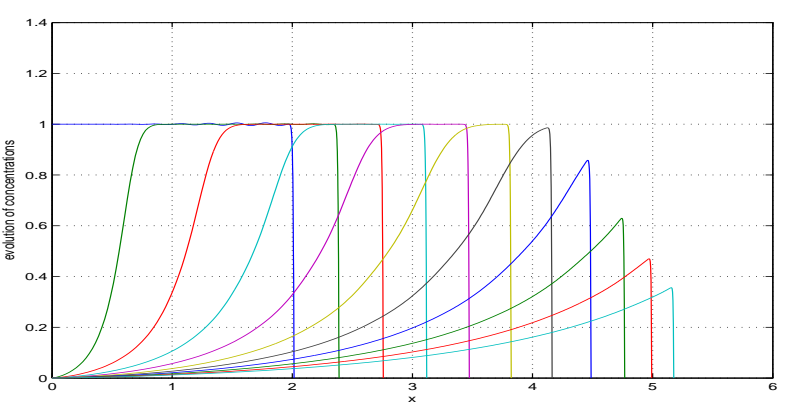

(b) Phase II, $t \in(4,12)$

Figure 3: Method II: $p=0.25, N=100, P e^{-1}=0.0025$

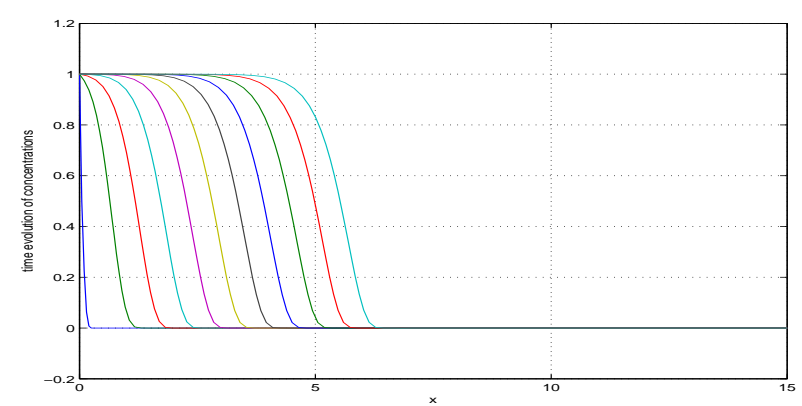

(a) Phase I, $t \in(0,8)$

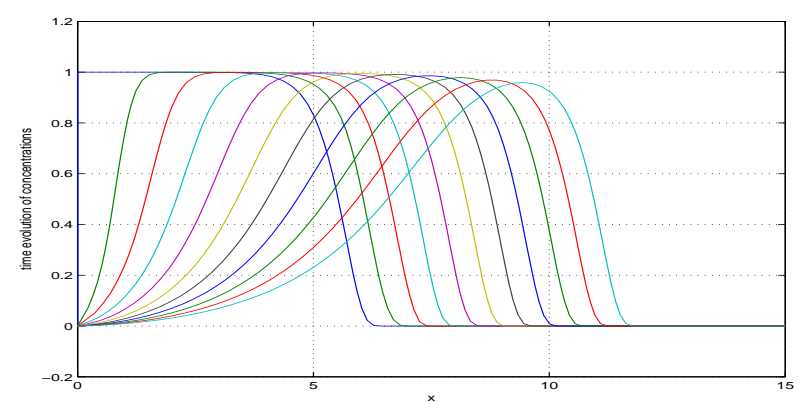

(b) Phase II, $t \in(8,16)$

Figure 4: Method I: Freundlich-Langmuir sorption isotherm $\Psi_{n}(s)=\frac{s^{0.5}}{1+s^{0.5}}$

is needed.

Now we present the solution of the more strongly degenerate problem, where instead of $P e^{-1}=0.05$ we take $P e^{-1}=0.0025$. This problem is very close to the nonlinear transport problem. The time evolution of phase $\mathrm{I}$ is presented in Fig. 3(a) and the time evolution of phase II is presented in Fig. 3(b). We notice that the initial state of the second phase is almost piecewise constant with acceptable front shock and unacceptable shock at $x=0$, which develops to the rarefaction wave. When this wave reaches the front, the solution decreases and the front shock proceeds with the Ranking-Hugoniot speed.

We present three additional results for different isotherms. In Fig. 4 we consider (21) with a generalized FreundlichLangmuir (F-L) sorption isotherm $\Psi_{n}(s)=\frac{s^{0.5}}{1+s^{0.5}}$. In Fig. 5 we present the solution of irreversible adsorption with Langmuir sorption isotherm $\Psi_{n}(s)=\frac{s}{1+s}$, where the adsorption process is modelled by

$$
\partial_{t} S=\kappa \max \left(0, \Psi_{n}(C)-S\right)
$$

Finally, in Fig. 6 we also present irreversible adsorption, but now for a Freundlich isotherm $\Psi_{n}(s)=s^{0.5}$.

Experiment 5. In this experiment we compare methods I and II for a high rate of non-equilibrium adsorption. We consider (2) with $P e^{-1}=0.05, \theta=1, \rho=1$ and $\Psi_{e}(s)=s^{0.5}, \Psi_{n}(s)=s^{0.5}, \kappa=15$. This represents adsorption in equilibrium and non-equilibrium mode. Since the non-equilibrium part of adsorption is close to adsorption in equilibrium mode, we can compare it with the adsorption in equilibrium mode with adsorption isotherm $\Psi_{e}(s)=2 s^{0.5}$. The adsorption in equilibrium/non-equilibrium mode is realised by means of method I in Fig. 7(a); the adsorption in equilibrium mode is realised by applying method II, see Fig. 7(b). These results can also be compared with those obtained by method I, where $\kappa=15, \Psi_{e}(s)=0$, and $\Psi_{n}(s)=2 s^{0.5}$. These results are presented in Fig. 7(c). As mentioned before, application of method I to equilibrium and also to equilibrium/non-equilibrium adsorption problems is limited by the stability problem of the ODE-solver in the case of degenerate adsorption in equilibrium mode (i.e., $\left.\Psi_{e}^{\prime}(0)=\infty\right)$, where some regularization is needed. Method II is substantially more efficient in that case because of 


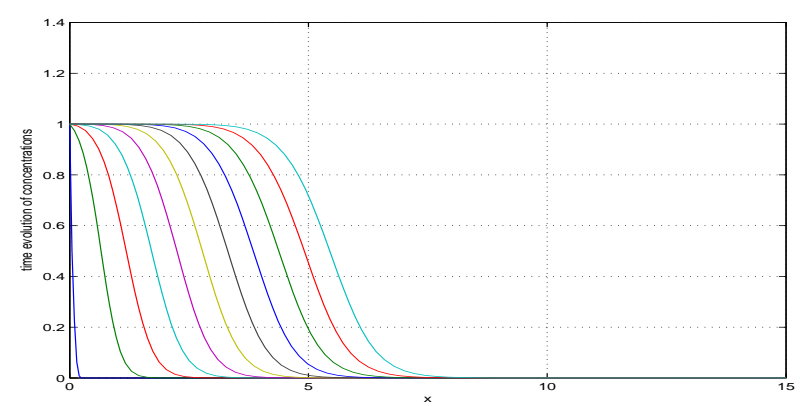

(a) Phase $\mathrm{I}, t \in(0,8)$

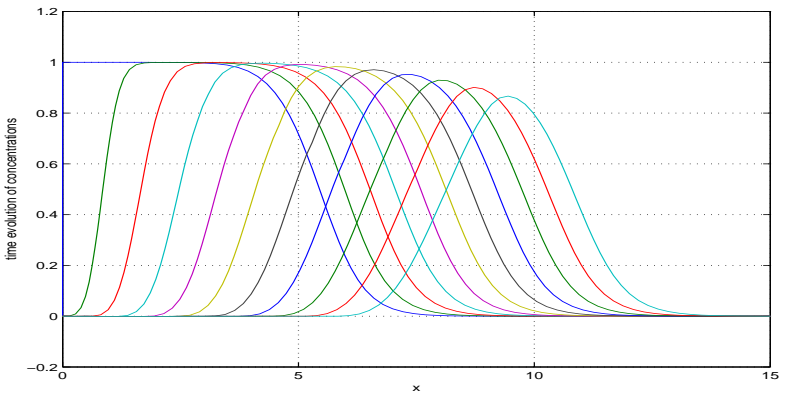

(b) Phase II, $t \in(8,16)$

Figure 5: Method I: irreversible Langmuir adsorption

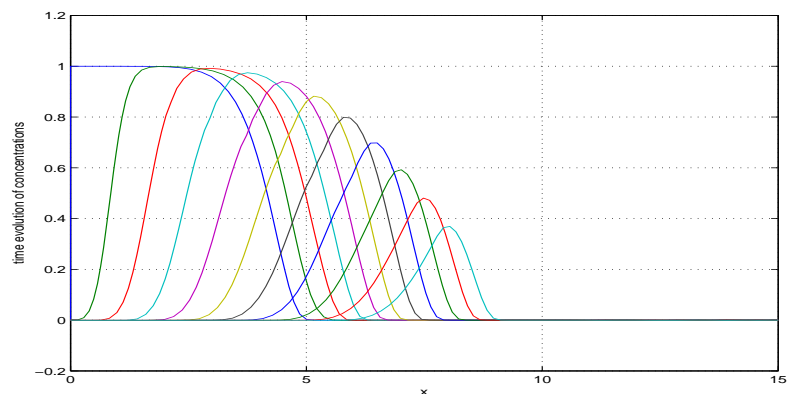

Figure 6: Method I: phase II, $t \in(8,16)$, irreversible Freundlich adsorption

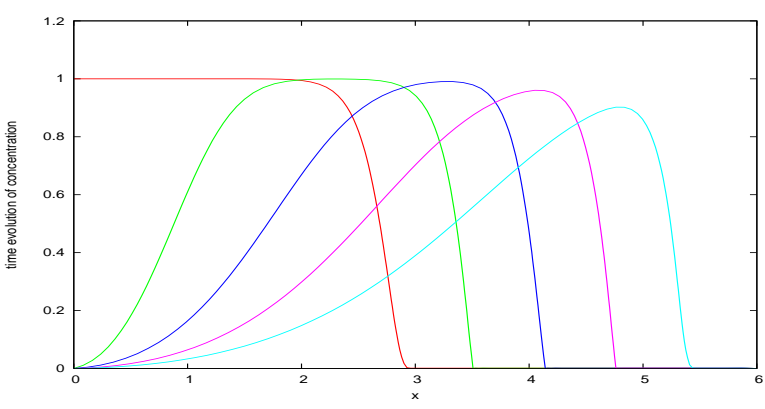

(a) Method I, equilibrium and non-equilibrium adsorption

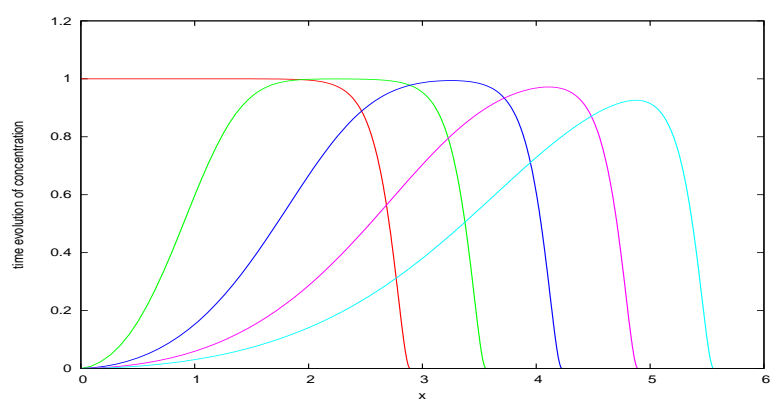

(b) Method II, equilibrium adsorption

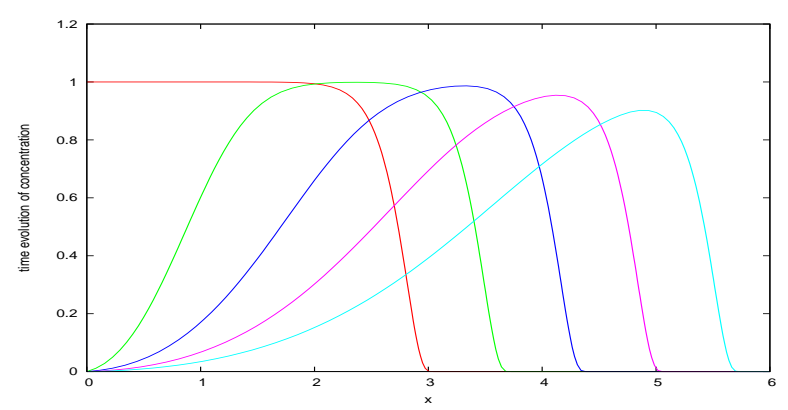

(c) Method I, non-equilibrium adsorption

Figure 7: Phase II, $t \in(8,16), 3$ realizations of high-rate adsorption at $t=8,10,12,14$ and 16 . 
the additional information from the interface model. This experiment shows that using method I with only a nonequilibrium high-rate adsorption is an alternative.

\section{Conclusion}

We have compared three methods for the solution of convection-diffusion-adsorption problems in 1D, based on dynamical discretization. In method I moving grid points were used, where the movement of the grid points was an integral part of the solution method. In method II a domain transformation to a fixed grid was used for the interface problem which arises for some types of equilibrium adsorption, whereas method III was equal to method I without the moving grid points.

When method II is applicable, then it is the best method, and should be preferred. Method III does not have the overhead of calculating the moving grid points making it normally less time consuming. However, for non-equilibrium adsorption the computing time increases dramatically. Although Method I is generally usable and gives excellent results in most cases, it needs unfeasibly many steps to converge when highly non-linear equilibrium adsorption is considered. This drawback can be overcome by considering high-rate $(\kappa$ large) non-equilibrium adsorption. Note that the conclusion that Method I is an improvement over Method III for non-equilibrium adsorption only, can only be made for the 1D example given here. It is a typical 1D phenomenon, as the computational cost of a denser grid in 1D might be acceptable, but this will normally not be the case in higher space dimensions.

For the type of experiments envisioned, a correct approximation of the interface is extremely important as it determines the shape of the break through curves measured. The experiments clearly show that even advanced dynamical discretizations have several issues in specific regimes. We have identified which technique should be used for which situation. Several techniques have been presented to overcome various difficulties encountered.

\section{Acknowledgments}

The first and last author were partially supported by Slovak Agency grant APVV-0351-07. The first author also obtained financial support from the BOF 2005/GOA-project of UGent (016A0405).

\section{References}

[1] J. Bear, Dynamics of Fluid in Porous Media, Elsevier, 1972.

[2] P. Knabner, F. Otto, Solute tansport in porous media with equilibrium and nonequilibrium multiple-site adsorption, Nonlinear Analysis 42 (2000) 381-403.

[3] N.-Z. Sun, Mathematical modeling of groundwater pollution, Springer-Verlag, 1996.

[4] D. Constales, J. Kačur, B. Malengier, A precise numerical scheme for contaminant transport in dual-well flow, Water Resources Research 39 (2003) 1303.

[5] C. Dawson, C. Van Duijn, M. Wheeler, Characteristic-Galerkin methods for contaminant transport with non-equilibriumadsorption kinetics, SIAM J.Numer.Anal 31 (1994) 982-999.

[6] J. Barrett, P. Knabner, An improved error bound for a Lagrange-Galerkin method for contaminant transport with non-Lipschitzian adsorption kinetics, SIAM J.Numer.Anal 35 (1998) 1862-1882.

[7] P. Knabner, C. Van Duijn, Solute tansport in porous media with equilibrium and non-equilibrium multiple-site adsorption: Traveling waves, Jounal für die reine und angewandte Mathematic 415 (1995) 1-49.

[8] J. Kačur, B. Malengier, M.Remešíková, Solution of contaminant transport with equilibrium and non-equilibrium adsorption, CMAME 194 (2005) 479-489.

[9] J. Kačur, M. R. ková, B. Malengier, Contaminant transport with adsorption and their inverse problems, Computing and Visualization in Science 10 (2007) 29-42.

[10] M. Remešíková, Solution of convection-diffusion problems with non-equilibrium adsorption, Journal of Computational and Applied Mathematics 169 (2004) 101-116.

[11] G. Kačurová, Numerical modelling of convection-diffusion-reaction problems with free boundary in 1D, arXiv preprint (arXiv:0909.0363).

[12] J. Verwer, J. Blom, R. Furzeland, P. Zegeling, A moving grid method for one-dimensional PDEs based method of lines, in: J.E.Flaherty, P.J.Paslon, M.S.Shephard, J.D.Vasilakis (Eds.), Adaptive methods for Partial Differential Equations, SIAM, Philadelphia, 160-175, 1989.

[13] G. Beckett, J. Mackenzie, M. Robertson, D. Sloan, On the numerical solution of one-dimensional PDEs using adaptive methods based on equidistribution, J. Comput. Phys. 167 (2001) 372-392.

[14] N. Toride, L. F.J., van Genuchten M.T., A comprehensive set of analytical solutions for non-equilibrium solute transport with first-order decay and zero-order production, Water Resources Research 29 (1993) 2167-2182. 